\title{
A semi-analytical, two-dimensional model for AIGaN/GaN high-electron-mobility-transistor Schottky currents at high reverse voltages
}

\author{
Rik Jos ${ }^{\text {a) }}$ \\ Microwave Electronics Laboratory, Chalmers University of Technology, Kemivägen 9, 41296 Gothenburg, \\ Sweden and NXP Semiconductors, Gerstweg 2, 6534AE Nijmegen, The Netherlands
}

(Received 18 July 2013; accepted 2 August 2013; published online 21 August 2013)

\begin{abstract}
A semi-analytical model is derived for the reverse Schottky gate current in AlGaN-GaN highelectron-mobility-transistors (HEMTs) in off-state at high drain voltage. At elevated drain voltages, the depletion of the two-dimensional electron gas (2-DEG) not only exists directly underneath the gate contact but also extends from the gate towards the drain. This increases the electric fields at the gate edge on the drain side, which causes an increase in the gate leakage current. In this paper, a new method is proposed to calculate the electric fields at the gate edge using conformal mapping of the charge configuration in the device. This method also allows calculation of the influence of charges trapped at the interface between the semiconductor and the passivation layer next to the gate. It is shown that direct tunneling is the dominant current mechanism at high drain voltages, such that the current becomes insensitive to temperature variation. Interface charges next to the gate exert a large influence on the high voltage leakage current. It is argued that temperature variations of the high voltage leakage current are caused by temperature dependent changes in trapped charge. (C) 2013 AIP Publishing LLC. [http://dx.doi.org/10.1063/1.4818832]
\end{abstract}

\section{INTRODUCTION}

AlGaN-GaN high-electron-mobility-transistors (HEMTs) have attracted much attention over the last decade for possible use in RF power applications like radar systems, cellular base station, and microwave transmitters. One of the important factors limiting the usability of these HEMTs is a high Schottky gate leakage current, especially when a high drain voltage is applied. Any gate current flowing outwards from the gate through a (parasitic) resistor somewhere in the gate path will cause a voltage increase on the internal gate of the transistor above the voltage that is externally applied. This gate selfbiasing effect will increase the drain current, which in turn will lead to more power dissipation in the HEMT, higher device temperature and therefore higher gate leakage current. This mechanism can cause bias run-away, which destroys the transistor. A low leakage current is therefore required to achieve rugged operation.

Although gate leakage has been studied in AlGaN-GaN HEMTs, the leakage mechanism is still not fully understood nor is it clear along which path the current flows in the device. Goswami et al. have proposed a model in which the electrons move from gate to drain by hopping between surface traps of the AlGaN layer at the interface with the device passivation. ${ }^{1}$ In this paper, however, we argue that the gate current is caused by electrons that transit through the AlGaN Schottky barrier between gate and 2-DEG and from there move to the drain via the 2-DEG. In our proposed model, the degree of depletion of the 2-DEG is crucial. At low bias, between threshold and zero Volt, the current can essentially be analyzed by a one-dimensional model as published before; ${ }^{2}$

a)rik.jos@nxp.com while at high bias, the problem is fundamentally twodimensional. The low bias case was used to derive a physical model for the current through the Schottky barrier. In this paper, we will use that physical model in a situation with high bias voltages. Conformal mapping techniques are used to calculate the two-dimensional electric fields in the device. Twodimensional analysis of gate leakage current has been done before $^{3,4}$ using TCAD simulations. However, these studies often use high reverse bias voltages (larger than the threshold voltage), which makes it hardly possible to determine the physics behind the current mechanism. An exception is ${ }^{5}$ in which, just as in our model, a clear distinction is made between mechanisms generating the current at reverse bias below and above threshold voltage. Usually, reasonable fits with measurements are obtained if charged traps are assumed to reside in various places in the device. However, this approach does not guarantee that such traps are actually present, since similar results can also be obtained by many other trap configurations. Since in this paper, we use a physical model for the current flow through the Schottky barrier that is verified and calibrated at low bias voltages, there are fewer degrees of freedom at high bias. In fact, we will only use the net surface charge of the AlGaN layer as variable.

In a previous paper, ${ }^{2}$ several models for the Schottky gate current were evaluated at low reverse voltages, ranging between the threshold voltage $V_{t}\left(V_{t}<0\right)$ and zero volt. It was argued that the dominant mechanism is current flow through defects in the Schottky barrier that have an abnormally low barrier height of $0.58 \mathrm{eV}$. However, these defects only form a fraction $\left(\sim 10^{-4}\right)$ of the total junction area. In this paper, we extend the analysis to the case of a high voltage on the drain, which increases the electric fields at the gate edge and causes Schottky current increase. We assume 
the 2-DEG under the gate is always fully depleted, which means that there is no drain current flowing except for part of the gate leakage current. Analytical expressions for the field in two dimensions near the gate edge are derived. The field is determined by the net charges in the 2-DEG under and next to the gate and by possible net charges in the semiconductor-passivation interface.

\section{GEOMETRY AND CONFORMAL MAPPING}

In Ref. 2, we only considered a two terminal device with a gate and a source. In this paper, a three terminal offstate HEMT is treated. However, the source is not used as an independent terminal but the gate source voltage is assumed to always be equal to the threshold voltage, such that the 2DEG under the gate is completely depleted. So while the source and gate potentials are tightly coupled, the Schottky gate current will be described as function of the drain voltage only. Conformal mapping ${ }^{6,7}$ is used to describe the gate geometry, the 2-DEG, and the interface layer between AlGaN and passivation. We assign zero potential to the gate (note that this implies a positive voltage $-V_{t}$ on the source) and assign a charge distribution in the 2-DEG and at the interface. From a given charge distribution, the potential everywhere in the device is calculated. This implies that the drain potential is a result of and not an input to the calculations. The charge distribution therefore has to fulfill certain conditions to achieve a physically valid solution. Details of the charge distribution will be described in Sec. III. Since conformal mapping works in the complex plane and we like to keep the mathematics as simple as possible, the gate is put in the lower right part of the complex plane. This plane will be denoted as the $z$-plane, where $z=(-x, y)$ or, equivalently, $z=-x+j y$ and $j=\sqrt{ }-1$. Fig. 1 shows the configuration of the gate and the 2-DEG. Note that this figure is rotated by $180^{\circ}$. This way the figure conforms to the regular way of displaying a FET's cross section. We have used $z=(-x, y)$ rather than $z=(x, y)$ such that the $x$-coordinate has positive values towards the right of the figure, as is the usual convention. To calculate the electric field, the dielectric constants of the passivation and AlGaN are assumed to be the same.

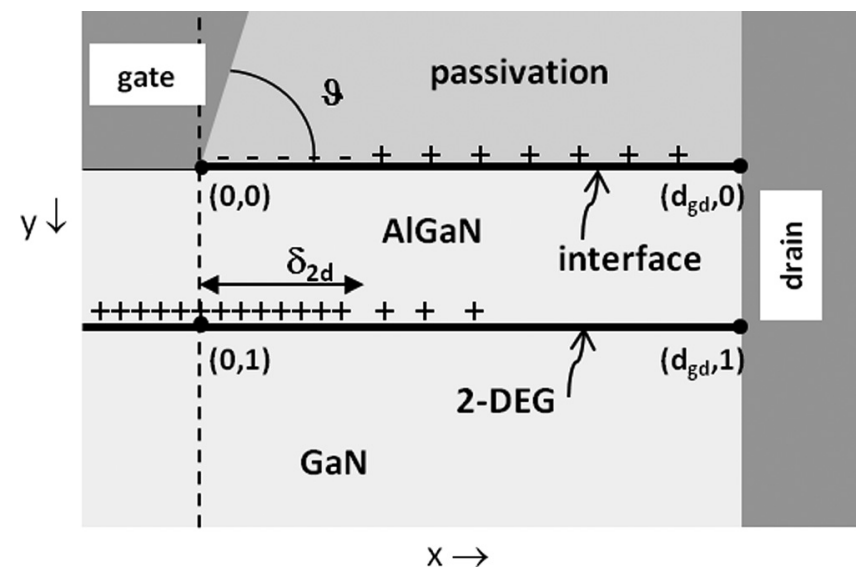

FIG. 1. The complex $z$-plane showing the gate, the 2-DEG, and the AlGaN to passivation interface. As an example, a possible configuration of net charges is shown in the 2-DEG and at the interface.
Since the actual values are usually quite comparable, this will only introduce a minor error in the calculated electrical potential. To simplify the mathematics further, we denote the co-ordinate of the gate edge by $(0,0)$ and we place the 2DEG at $(x, 1)$. Note that this choice defines all distances in the structure in units of the AlGaN layer thickness. The interface between $\mathrm{AlGaN}$ and passivation is placed at $(x, 0)$ for $x>0$. On the right side, the 2-DEG and the interface both end at the drain contact, which is placed at $\left(d_{g d}, y\right)$. The angle between gate edge and interface, which is determined by the gate processing, is denoted by $\vartheta$. Note that the gate height in this model is infinite, which is of course not realistic. In real devices, the gate shape and possible field plates will influence the electric fields in the device. However, since the gate current is mainly determined by electric fields very close to the gate corner, our model represents the relevant physical mechanisms that generate the current.

The complex $z$-plane is mapped onto another complex plane: the $\boldsymbol{w}$-plane, where $\boldsymbol{w}=(-u, v)$ or $\boldsymbol{w}=-u+j v$ by a very simple conformal mapping

$$
w=z^{\alpha} .
$$

Herein, $\alpha=\frac{\pi}{\pi+\vartheta}$.

Fig. 2 shows the gate, the 2-DEG, and the interface in the $\boldsymbol{w}$-plane. Note that also $\boldsymbol{w}=(-u, v)$ is used rather than $\boldsymbol{w}=(u, v)$, such that the $u$-coordinate obtains positive values towards the right in Fig. 2. The gate has been transformed into a half plane $(u, v)$ with $v<0$. The two dimensional electrical potential in the $\boldsymbol{w}$-plane can be solved for any charge distribution in the lower half plane. In the real threedimensional transistor, the charges in the 2-DEG and at the interface are sheet charges. These can be represented by a distribution of line charges. Since the complex plane is a two-dimensional cross section of the three-dimensional structure, every charge that is placed in Fig. 2 represents a line charge. Every line charge in the lower half of the $w$-plane contributes to the electrical potential by ${ }^{8}$

$$
\begin{aligned}
\Psi\left(u_{0}, v_{0}\right)= & \frac{-q}{2 \pi \varepsilon} \ln \left[\left(u_{0}, v_{0}\right)-(u, v)\right] \\
& +\frac{q}{2 \pi \varepsilon} \ln \left[\left(u_{0}, v_{0}\right)-(u,-v)\right]
\end{aligned}
$$

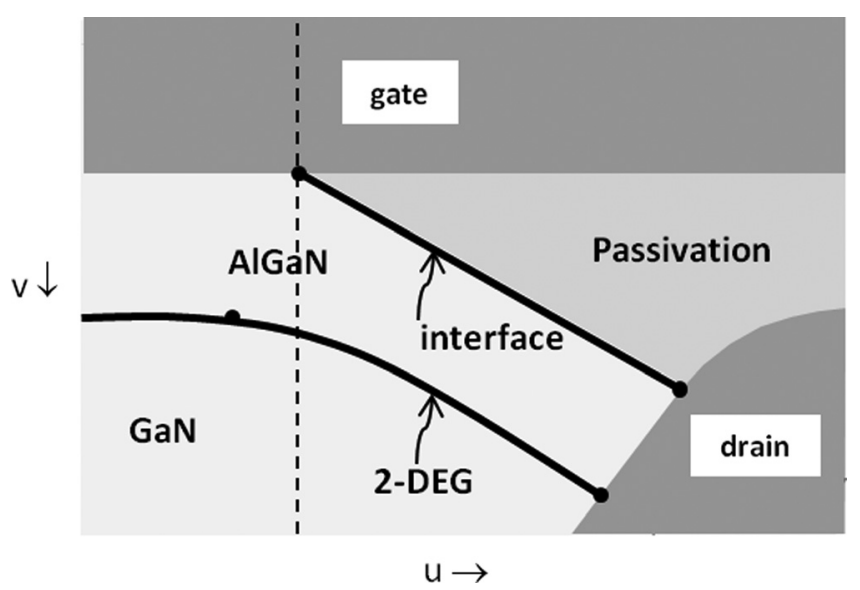

FIG. 2. The complex $w$-plane as a transformation of the $z$-plane of Fig. 1. 
or equivalently by

$$
\Psi\left(u_{0}, v_{0}\right)=\frac{-q}{2 \pi \varepsilon} \ln \left(\frac{r e^{i \varphi}}{r^{\prime} e^{i \varphi \prime}}\right)=\frac{-q}{2 \pi \varepsilon} \ln \left(\frac{r}{r^{\prime}}\right)+\frac{-q}{2 \pi \varepsilon} i\left(\varphi-\varphi^{\prime}\right) .
$$

This is the potential contribution at $\left(u_{0}, v_{0}\right)$ caused by a positive line charge $q$ (in Coulomb per meter in the direction perpendicular to the complex plane) at $(u, v)$, see Fig. 3. Note that (1) is the so-called complex potential. The real part describes the equipotential lines, while the imaginary part describes the flow lines that are perpendicular to the equipotential lines.

The transformation $\boldsymbol{w}=\boldsymbol{z}^{\alpha}$ can be worked out in terms of the co-ordinates $x_{0}, y_{0}$ and $u_{0}, v_{0}$ as

$$
\begin{aligned}
& u_{0}=-\left(x_{0}^{2}+y_{0}^{2}\right)^{\alpha / 2} \cos \left[\alpha \operatorname{atan}\left(\frac{y_{0}}{-x_{0}}\right)\right], \\
& v_{0}=\left(x_{0}^{2}+y_{0}^{2}\right)^{\alpha / 2} \sin \left[\alpha \operatorname{atan}\left(\frac{y_{0}}{-x_{0}}\right)\right] .
\end{aligned}
$$

Of course, exactly identical relations exists between $x, y$ and $u, v$.

In case a charge is located in the 2-DEG, $y=1$ in the $z$ plane and therefore these relations reduce to

$$
\begin{aligned}
& u=-\left(x^{2}+1\right)^{\alpha / 2} \cos [\alpha \operatorname{atan}(-1 / x)], \\
& v=\left(x^{2}+1\right)^{\alpha / 2} \sin [\alpha \operatorname{atan}(-1 / x)] .
\end{aligned}
$$

For charges at the interface, $y=0$ and $(2 \mathrm{a})$ reduces to

$$
\begin{aligned}
& u=-|x|^{\alpha} \cos (\alpha \pi), \\
& v=|x|^{\alpha} \sin (\alpha \pi) .
\end{aligned}
$$

Calculation of the potential in the $z$-plane is very easily done by substituting (2) into (1).

Next, we may replace $q$ in (1) by the infinitesimal charge $\rho d x$, which gives us the potential $\Psi\left(x_{0}, y_{0}\right) d x$ in the $z$-plane as a result of a certain line charge density $\rho$ located at $(x, y)$. Now, $\Psi\left(x_{0}, y_{0}\right) d x$ is only depending on parameters in the $z$-plane. Therefore, knowledge of the charge distribution in the $\boldsymbol{w}$-plane is not required, but $\Psi\left(x_{0}, y_{0}\right) d x$ can directly be

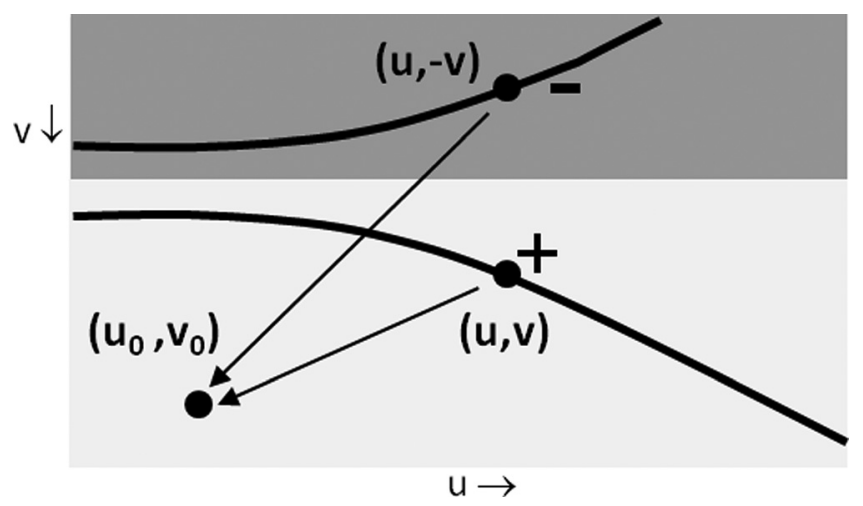

FIG. 3. The potential at $\left(u_{0}, v_{0}\right)$ caused by a positive charge at $(u, v)$ in the $\boldsymbol{w}$ plane. The influence of the gate is taken into account by the negative mirror charge at $(u,-v)$. integrated using the charge densities in the $z$-plane. The integration over the interface charges runs from 0 to $d_{g d}$, while the 2-DEG charges are integrated from a position $x \ll 0$, far enough under the gate where the electric field can be considered as one dimensional, to $d_{g d}$.

\section{CHARGE CONFIGURATION}

\section{A. 2-DEG charges}

If a large drain voltage is applied, the 2-DEG will deplete next to the gate on the drain side. Let us assume that full depletion is achieved over a certain distance $\delta_{2 d}$ next to the gate (see Fig. 1). Also full depletion is assumed under the gate, which would correspond to a situation where the source to gate bias just equals the threshold voltage. So the 2-DEG is fully depleted everywhere for $x<\delta_{2 d}$. For $x>\delta_{2 d}$, the 2-DEG will only be partly depleted, which implies that there are mobile electrons in the 2-DEG and the electric field must therefore be zero to avoid current flow in a static situation. Starting from $x=\delta_{2 d}$, the electron concentration rises gradually towards the drain. Since there is no self-consistent equation that describes the electron concentration in the 2-DEG, we will model the net charge density in the 2-DEG by an empirical equation

$$
\begin{array}{ll}
\text { for } \mathrm{x}<\delta_{2 d}: & \rho_{2 d}=q N \\
\text { for } \mathrm{x}>\delta_{2 d}: & \rho_{2 d}(x)=\frac{q N}{\left(1-\delta_{2 d}+x\right)^{\lambda}},
\end{array}
$$

where $\lambda$ is a fitting parameter and $q N$ is the concentration of positive charge in the 2-DEG when it is fully depleted. As can be seen from (3), the 2-DEG is fully depleted and has maximum positive charge for $x<\delta_{2 d}$. For $x>\delta_{2 d}$, the electron concentration rises and compensates part of the positive 2-DEG charge, reducing the total net charge. Also other functions, like an exponential function, can be used to describe the 2-DEG charge density. After charge optimization (see below), such functions will all describe almost the same charge distribution. We have chosen the equation in (3) because it only has one fitting parameter (since $\delta_{2 d}$ and $N$ are given) and it gives a slightly better optimization than an exponential function with also just one fitting parameter. Although the 2-DEG between gate and drain contains electrons as well as positive charges from the AlGaN polarization, we found that the net 2-DEG charge is always positive everywhere between gate and drain in all the cases that we calculated, meaning that the electron concentration is always less than the positive charge concentration in the 2-DEG.

\section{B. Drain contact}

Using conformal mapping, we cannot define a drain contact and apply a voltage boundary condition to the edge of that contact, like we did with the gate. Therefore, we define a charge at the position of the drain in such way that the appropriate boundary conditions for the drain contact are fulfilled. A charge $N_{d}$ is assigned to the 2-DEG at the position of the drain contact, i.e., at $(x, 1)$ with $x>d_{g d}$, the distance between gate and drain. It turns out that the electrical potential near the gate edge hardly depends on the exact size of 
the drain charge, since $N_{d}$ serves as a fitting parameter. Therefore, we can simply distribute it over a certain distance beyond $d_{g d}$. We took very arbitrarily a distance of $20 \mathrm{AlGaN}$ thickness units, so $N_{d}$ is distributed uniformly between $d_{g d}<x<d_{g d}+20$.

\section{Interface charges}

There are also net charges outside the 2-DEG, like polarization charges and charged donors, acceptors, and traps. ${ }^{9-11}$ The most important net charges outside the 2-DEG are located at the interface between the $\mathrm{AlGaN}$ and the passivation, due to the AlGaN polarization and surface traps. In our model, we will assume that the only charge outside the 2DEG is located at the interface. This means that we ignore any net charge in the $\mathrm{AlGaN}$ and GaN bulk as well as at the GaN to substrate interface. From theoretical calculations ${ }^{12}$ for an $\mathrm{Al}$ percentage of $18 \%$ and an $\mathrm{AlGaN}$ thickness of $25 \mathrm{~nm}$, we find: $N=1.0 \cdot 10^{13} \mathrm{~cm}^{-2}$. The equilibrium electron concentration in the 2-DEG is calculated to be ${ }^{12}$ $n_{0}=6.8 \cdot 10^{12} \mathrm{~cm}^{-2}$, which agrees well with Hall measurements of the electron concentration that vary between 6 and $7 \cdot 10^{12} \mathrm{~cm}^{-2}$. In equilibrium, i.e., far from any contact, the material must be neutral. So the net 2-DEG charge which is $q\left(N-n_{0}\right)$ must be compensated by an exactly opposite interface charge $-q N_{i f}$. Hence, in the absence of other charges, $N_{i f}=3.2 \cdot 10^{12} \mathrm{~cm}^{-2}$. It is reasonable to assume that this is also the interface charge between the gate and drain contacts.

\section{Charge distribution optimization}

The potential in the 2-DEG underneath the gate has a positive value of $-V_{T}$. Next to the gate, the potential rises in the region where the $2-\mathrm{DEG}$ is fully depleted and has to become constant for $x>\delta_{2 d}$, since the electric field is zero in that region. This constant value must equal the drain voltage. First, we start with assigning a net interface charge density between gate and drain. This could, for example, be given by a uniform density $-q N_{i f}$ everywhere. Next, we take a value for $\delta_{2 d}$. Then, there are only 2 fitting parameters, $\lambda$ and $N_{d}$, that need to be optimized such that the electric field becomes zero for $x>\delta_{2 d}$. This ensures that the charge distribution is physically sound. Once that is done, the drain voltage is obtained by averaging the potential, i.e., $\operatorname{Re}[\Psi(x, 1)]$, over the region between $\delta_{2 d}$ and drain contact. Of course, optimization of $\lambda$ and $N_{d}$ can best be done by a simple routine optimizing the error between $\operatorname{Re}[\Psi(x, 1)]$ and the obtained drain voltage. Fig. 4 shows examples of the potential $\operatorname{Re}[\Psi(x, 1)]$ in the 2-DEG for the HEMT structure. The gate angle was set to $\vartheta=45^{\circ}$. The gate edge is located at $x=0$. The AlGaN layer thickness is $25 \mathrm{~nm}$, and the gate to drain distance is $1.5 \mu \mathrm{m}$, which corresponds to $x=60$. The drain voltages and their errors are calculated as

$$
\begin{aligned}
V_{d} & =\frac{1}{d_{g d}-\delta_{2 d}} \int_{\delta_{2 d}}^{d_{g d}} \operatorname{Re}[\Psi(x, 1)] d x \\
\text { error }^{2} & =\frac{1}{d_{g d}-\delta_{2 d}} \int_{\delta_{2 d}}^{d_{g d}}\left\{V_{d}-\operatorname{Re}[\Psi(x, 1)]\right\}^{2} d x .
\end{aligned}
$$

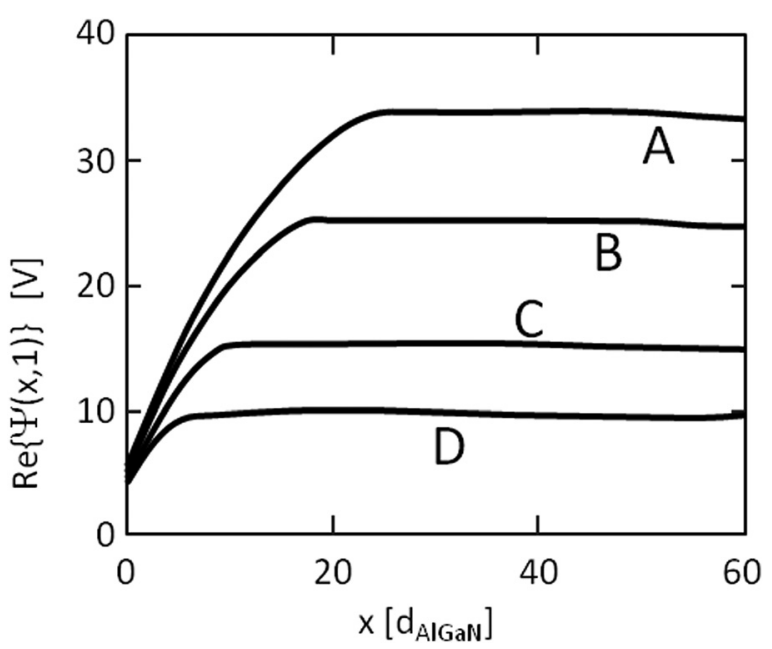

FIG. 4. Real part of the 2-DEG potential between gate and drain in a transistor example for 4 different fitting parameter settings: Curve A: $\delta_{2 d}=25$, $\lambda=0.16, N_{d}=7 \cdot 10^{17} \mathrm{~cm}^{-1}$ give $V_{d}=33.70 \pm 0.19 \mathrm{~V}$, Curve B: $\delta_{2 d}=18$, $\lambda=0.175, N_{d}=5.3 \cdot 10^{17} \mathrm{~cm}^{-1}$ give $V_{d}=24.95 \pm 0.16 \mathrm{~V}$, Curve C: $\delta_{2 d}=10$, $\lambda=0.22, N_{d}=3.5 \cdot 10^{17} \mathrm{~cm}^{-1}$ give $V_{d}=15.01 \pm 0.16 \mathrm{~V}$, Curve D: $\delta_{2 d}=5$, $\lambda=0.25$, and $N_{d}=2.710^{17} \mathrm{~cm}^{-1}$ give $V_{d}=9.5 \pm 0.2 \mathrm{~V}$.

The parameters are optimized till the error is minimal.

Fig. 5 shows the equipotential lines $\operatorname{Re}(\Psi)$ and the flow lines $\operatorname{Im}(\Psi)$ at the gate edge for the example transistor at a drain voltage of $24.95 \mathrm{~V}$. Note that the $2-\mathrm{DEG}$ is not visible in these figures since the maximum $y$-value is 0.5 . The dotted area in the upper right corner denotes the passivation layer, which we assume to be an isolator. So the flow lines passing through that region do not carry any current.

\section{TWO-DIMENSIONAL GATE CURRENT}

In Sec. III, we derived a method to calculate the twodimensional electric field and associated flow lines near the

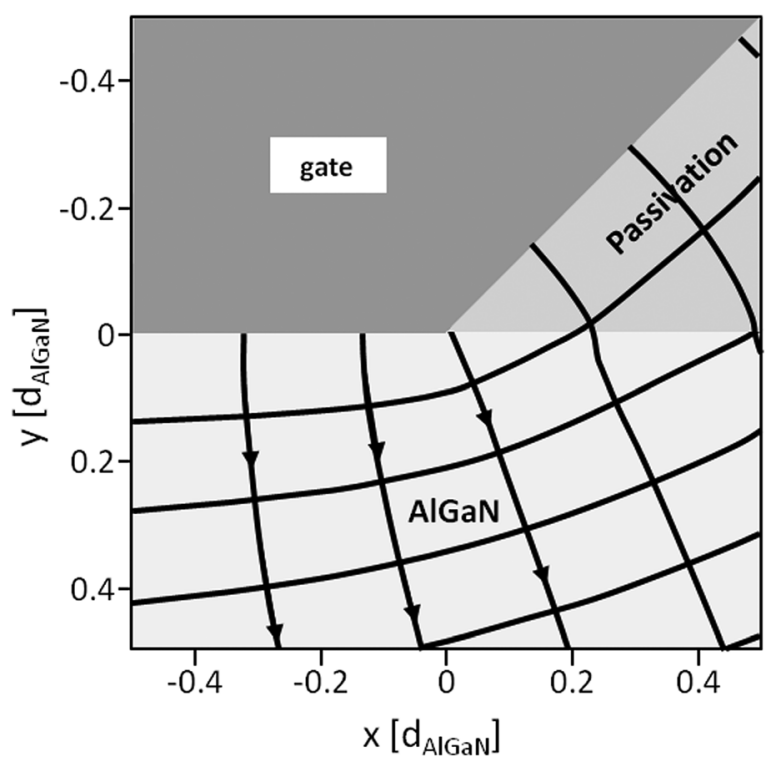

FIG. 5. Equipotential and flow lines at $24.95 \mathrm{~V}$. The gate contact is indicated by the dark shaded area. The passivation is shown by the transparent lightly shaded area. Current flow is indicated by arrows, while the equipotential lines are perpendicular to the flow lines. Note that, although flow lines are defined in the passivation, no current flows through it. 
gate edge. Our aim in this section is to develop a method for calculating the gate current that flows near the gate edge. The current will flow along the flow lines (Fig. 5) that originate at the gate metal contact to the $\mathrm{AlGaN}$, so at $(x, 0)$ where $x<0$. It is assumed that there is no current flowing through the passivation. The electric field $E$ is always directed tangential to the flow lines. If we parameterize a flow line by parameter $s$, we have

$$
\vec{\nabla} \cdot \vec{E}=\frac{d E}{d s}=\frac{\rho}{\varepsilon} .
$$

In the absence of charge, as is assumed between the gate and the 2-DEG, $\rho=0$ and hence $E$ is constant along the flow lines. This means that the electrons from the gate experience a triangular barrier that is described by the vertical electric field $E_{y}(x, 0)$ at the edge of the gate contact

$$
E_{y}(x, 0)=-\left.\frac{d \operatorname{Re}[\Psi(x, y)]}{d y}\right|_{y=0}
$$

This field can be calculated directly from the expressions for the potential as given in the Appendix. The 2-DEG contribution to the $E$-field, $E_{2 d}$, is given by

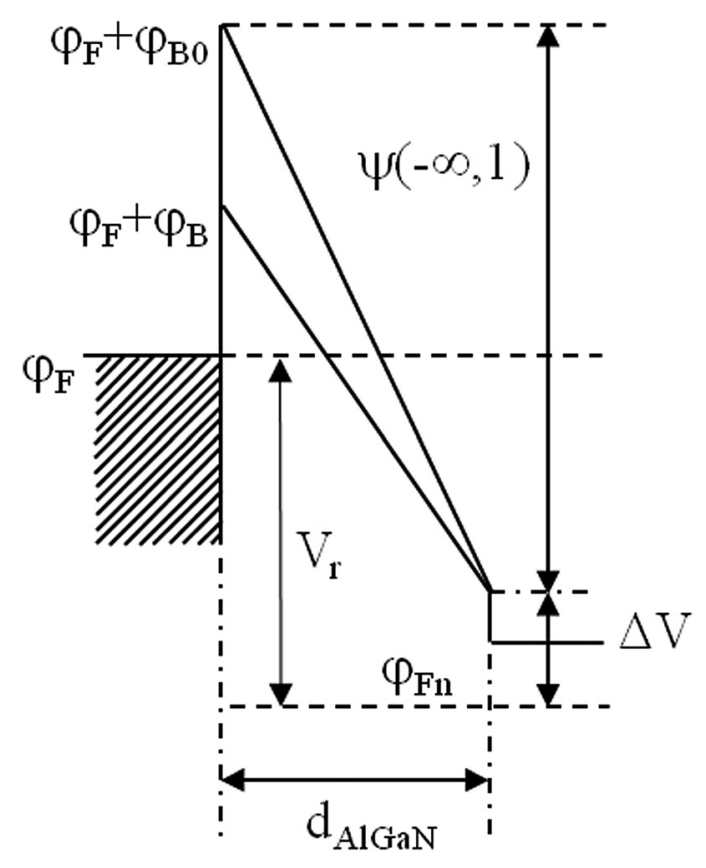

FIG. 6. Potential underneath the gate far from the gate edge when the 2DEG is fully depleted. The height of the intact barrier $\varphi_{B 0}$ relates directly to the 2-DEG sheet charge concentration. The current flows through defects with lower barrier height $\varphi_{B}$.

$$
E_{2 d}(x, 0)=\frac{q}{2 \pi \varepsilon} \int_{\Delta}^{d_{g d}} \frac{\rho_{2 d}(t)(-x)^{\alpha-1} 2 \alpha\left(t^{2}+1\right)^{\alpha / 2} \sin [\alpha \operatorname{atan}(-1 / t)]}{\left\{(-x)^{\alpha}-\left(t^{2}+1\right)^{\alpha / 2} \cos [\alpha \operatorname{atan}(-1 / t)]\right\}^{2}+\left(t^{2}+1\right)^{\alpha} \sin ^{2}[\alpha \operatorname{atan}(-1 / t)]} d t .
$$

The interface charge contribution, $E_{i f}$, is

$$
E_{i f}(x, 0)=\frac{q}{2 \pi \varepsilon} \int_{0}^{d_{g d}} \frac{\rho_{i f}(t)(-x)^{\alpha-1} 2 \alpha t^{\alpha} \sin (\alpha \pi)}{\left\{(-x)^{\alpha}-t^{\alpha} \cos (\alpha \pi)\right\}^{2}+(-t)^{2 \alpha} \sin ^{2}(\alpha \pi)} d t .
$$

The contribution from the drain contact, $E_{d}$, can be directly found modifying (5)

$$
E_{d}(x, 0)=\frac{q N_{d}}{2 \pi \varepsilon \Delta_{d}} \int_{d_{g d}}^{d_{g d}+\Delta_{d}} \frac{(-x)^{\alpha-1} 2 \alpha\left(t^{2}+1\right)^{\alpha / 2} \sin [\alpha \operatorname{atan}(-1 / t)]}{\left\{(-x)^{\alpha}-\left(t^{2}+1\right)^{\alpha / 2} \cos [\alpha \operatorname{atan}(-1 / t)]\right\}^{2}+\left(t^{2}+1\right)^{\alpha} \sin ^{2}[\alpha \operatorname{atan}(-1 / t)]} d t .
$$

In Ref. 2, it was reported that the Schottky current is flowing through defects that locally lower the junction barrier height. In such defects, the electric field is lower than what is calculated via (4). In Fig. 6, we assume a fully depleted 2-DEG and a triangular barrier. The height of the intact barrier is defined as $\varphi_{B O}$, and the barrier height in the defects as $\varphi_{B}$. The electric field of the intact barrier is given as $^{2}$

$$
E_{0}=-\frac{\varphi_{B 0}+V_{r}-\Delta V}{d_{A l G a N}}
$$

where $V_{r}$ is the reverse Schottky bias and $\Delta V$ is the sum of the conduction band offset between $\mathrm{AlGaN}$ and $\mathrm{GaN}$ and the GaN flatband voltage. See Fig. 6.

In the two-dimensional model, in which the 2-DEG is fully depleted, the potential under the gate, far away from the gate edge is given by $\Psi(-\infty, y)$, and hence

$$
\Psi(-\infty, 1)=\varphi_{B 0}+V_{r}-\Delta V .
$$

Note that, assuming a triangular barrier, there is a direct relation between $N$ and $\varphi_{B 0}$, since 


$$
\Psi(-\infty, 1)=\frac{q N d_{A l G a N}}{\varepsilon}
$$

Using $d_{A l G a N}=25 \mathrm{~nm}, N=1.0 \cdot 10^{13} \mathrm{~cm}^{-2}, V_{r}=-2.5 \mathrm{~V}$, and $\Delta V=-0.4 \mathrm{~V}$, we find $\varphi_{B 0}=2.656 \mathrm{eV}$. In two dimensions, the electric field in the defects can be calculated by scaling $E_{2 d}, E_{i f}$, and $E_{d}$ to $E_{2 d}^{\prime}, E_{i f}^{\prime}$, and $E_{d}^{\prime}$, respectively, by

$$
\begin{aligned}
E^{\prime}{ }_{2 d} & =\varepsilon \frac{\varphi_{B}+V_{r}-\Delta V}{q N d_{A l G a N}} E_{2 d}, \\
E^{\prime}{ }_{i f} & =\varepsilon \frac{\varphi_{B}+V_{r}-\Delta V}{q N d_{A l G a N}} E_{i f}, \\
E_{d}^{\prime} & =\varepsilon \frac{\varphi_{B}+V_{r}-\Delta V}{q N d_{A l G a N}} E_{d} .
\end{aligned}
$$

In Ref. 2, it was shown that the current density, $J(x)$, can be modeled assuming contributions by tunneling, thermionic field emission, and thermionic emission through and over a triangular barrier

$$
\begin{aligned}
& J(x)=\frac{4 \pi m_{m} q^{3}}{h^{3}} \int_{0}^{\varphi_{F}+\varphi_{B} \varphi_{F}+\varphi_{B}-\varphi_{y}} \int_{0}^{\alpha_{s}} \\
& \times \frac{\exp \left\{-\frac{\alpha_{s}}{E_{T O T}(x)}\left(\varphi_{F}+\varphi_{B}-\phi_{\text {tun }}\right)^{3 / 2}\right\}}{1+\exp \left\{\frac{q\left(\phi_{y}+\phi_{/ /}-\varphi_{F}\right)}{k T}\right\}} d \phi_{/ /} d \phi_{y} \\
& +\frac{4 \pi m_{m} q k^{2} T^{2}}{h^{3}} \exp \left(\frac{-q \varphi_{B}}{k T}\right) \text {. }
\end{aligned}
$$

The meaning of the symbols: $m_{m}$ is the electron mass in the metal, $\varphi_{F}$ is the metal Fermi energy, $\varphi_{B}$ is the barrier height, $\phi_{y}$ is the electron energy in the metal in the $y$-direction towards the junction: $\phi_{y}=\hbar^{2} k_{y}{ }^{2} / 2 m_{m}, \varphi_{/ /}$is the electron energy in the metal parallel to the junction: $\phi_{/ /}=\hbar^{2} k_{x}^{2} /$ $2 m_{m}+\hbar^{2} k_{z}^{2} / 2 m_{m}$ and $\phi_{\text {tun }}$ is the part of the electron energy available for tunneling: $\phi_{t u n}=\phi_{y}+\phi_{/ /}\left(m_{s}-m_{m}\right) / m_{s}$, where $m_{s}$ is the electron mass in the AlGaN and $\hbar=h / 2 \pi$ is Planck's constant. Furthermore,

$$
\alpha_{s}=\frac{4 \sqrt{2 m_{s} q}}{3 \hbar} .
$$

The total Schottky current can now be found by adding the contributions in (8) to: $E_{\text {tot }}(x)=E_{2 d}^{\prime}(x, 0)+E_{i f}^{\prime}(x, 0)$ $+E_{d}^{\prime}(x, 0)$, insert $E_{t o t}(x)$ in (9) and integrate over the Schottky contact area. Although this procedure would give an analytical expression for the Schottky current, the equation would become intractable. Therefore, we use a more numerical approach. The gate contact surface is divided in the $x$-direction into small segments with widths $\Delta x_{i}$. Note that the $\Delta x_{i}$ do not need to be of equal size, so we take very small segments widths close to the gate edge and larger widths for segments further under the gate. $E_{T O T, i}$ and the current density $J_{i}$ are both calculated per segment. The total gate current is obtained by $I=W \sum J_{i} \Delta x_{i}$, where $W$ is the total gate width of the HEMT.

\section{MEASUREMENTS}

Measurements have been done on the AlGaN-GaN HEMT described at Fig. 4 with a total gate width of $2.4 \mathrm{~mm}$ by measuring the gate current as function of gate-drain voltage with open source terminal. The Schottky current was measured in the gate-drain voltage range between $-60 \mathrm{~V}$ and $0 \mathrm{~V}$ and at temperatures of $25^{\circ} \mathrm{C}, 75^{\circ} \mathrm{C}, 125^{\circ} \mathrm{C}$, and $175^{\circ} \mathrm{C}$. To see the influence of voltage stress on the GaN HEMT, the current-voltage characteristics were measured from $0 \mathrm{~V}$ to $-60 \mathrm{~V}$ and back from $-60 \mathrm{~V}$ to $0 \mathrm{~V}$. Both curves did not coincide fully between $-20 \mathrm{~V}$ and threshold voltage, especially at lower temperatures. The current is less when measured from $0 \mathrm{~V}$ to $-60 \mathrm{~V}$ than when measured from $-60 \mathrm{~V}$ to $0 \mathrm{~V}$, see Fig. 7.

To investigate the influence of temperature stress, the current voltage characteristics at $25^{\circ} \mathrm{C}$ were measured once more after the measurements at elevated temperature of Fig. 7. Measurements before and after this temperature stress are shown in Fig. 8.

Both in Fig. 7 as in Fig. 8, a kink in the I-V curves can be observed at $-2.5 \mathrm{~V}$, which is the threshold voltage of the HEMT. At high reverse voltages, i.e., below $-2.5 \mathrm{~V}$, the 2-DEG depletes next to the gate such that the additional voltage drops in the region between gate and drain. This is a strong indication that the leakage current is not caused by electron-hopping along the interface. Note that the current between threshold voltage and $0 \mathrm{~V}$ is not influenced by the voltage and temperature stresses. Since the current in this range is determined by the intrinsic gate contact as described in Ref. 2, we conclude that the intrinsic Schottky contact is not affected.

This low voltage current can very well be calculated by the model of Ref. 2 if we assume defects in the Schottky barrier that have a reduced barrier height of $0.58 \mathrm{eV}$ and a total surface area that is a fraction $6 \times 10^{-4}$ of the total Schottky
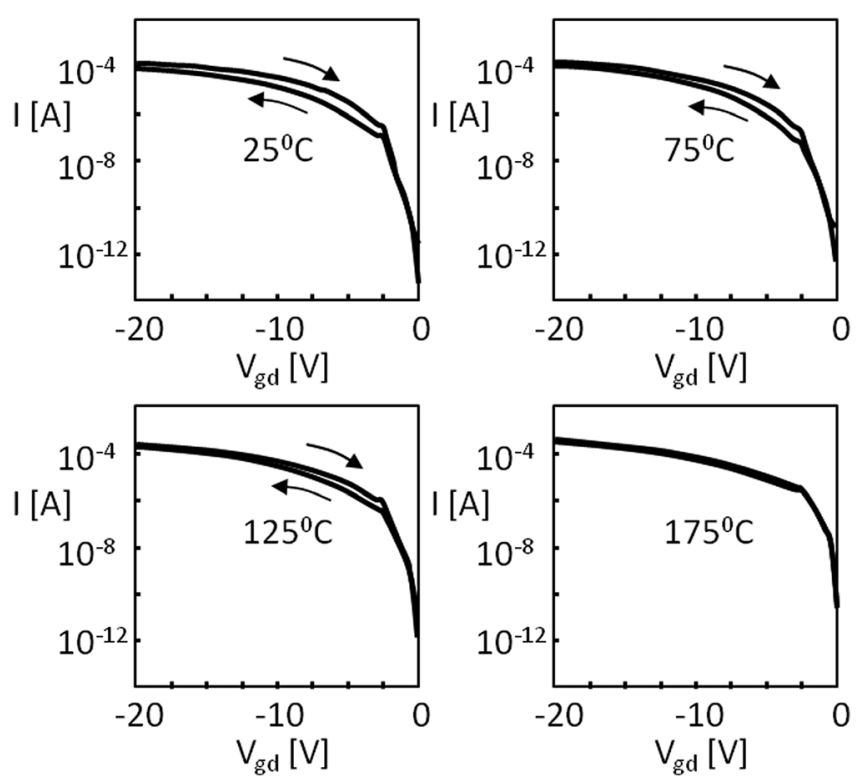

FIG. 7. Schottky current vs. gate-drain voltage for several temperatures, measured from 0 to $-60 \mathrm{~V}$ (lower curves) and back (higher curves). Only the voltage range between -20 and $0 \mathrm{~V}$ is displayed to show the hysteresis. 


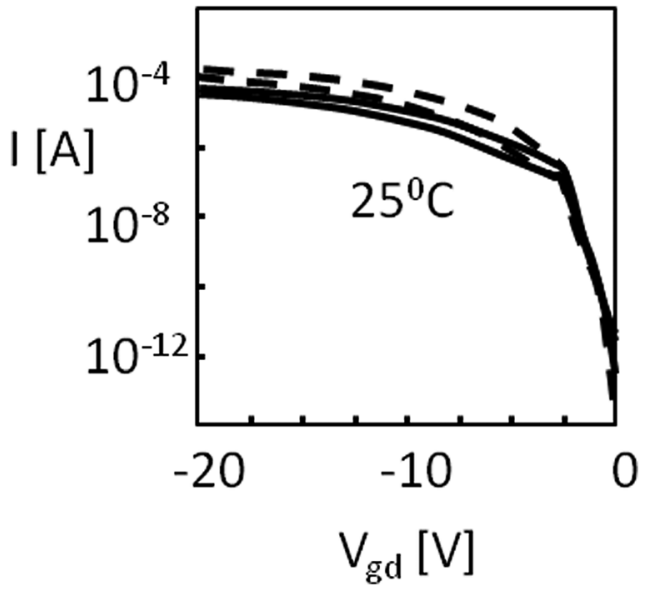

FIG. 8. Gate current vs. gate-drain voltage, measured from 0 to $-60 \mathrm{~V}$ and back, at $25^{\circ} \mathrm{C}$ before (solid lines) and after (dotted lines) the measurements at higher temperatures, indicating the device changes due to stresses during these higher temperature measurements.

area. Note that the barrier height is equal to that found on the transistor in Ref. 2, while the total surface area of the defects is 3 times larger.

The high voltage current is calculated using (9) for a transistor in which the negative interface charge density is $N_{i f}=3.2 \cdot 10^{12} \mathrm{~cm}^{-2}$ and the positive sheet charge density is $N=10^{13} \mathrm{~cm}^{-2}$. The results are shown in Fig. 9. It is clear that the assumed charge configuration, which was based on theoretical calculations, cannot explain the observed current.

The measurements as well as the calculations in Fig. 9 show that at very high reverse voltages, all results converge and become nearly independent of temperature and voltage. Our calculations show that under these conditions, the current is for a large part dominated by Fowler-Nordheim tunneling through the reduced barrier in the defects. Any residual temperature dependence at high reverse voltages can be explained by changes in the interface charge density $N_{\text {if }}$ due to temperature dependent occupancy of interface traps.

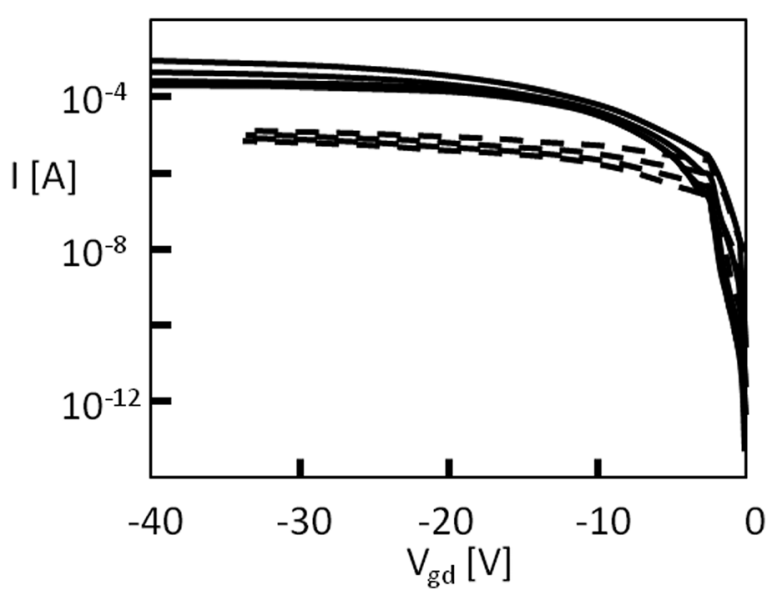

FIG. 9. Schottky current vs. gate-drain voltage for $25^{\circ} \mathrm{C}, 75^{\circ} \mathrm{C}, 125^{\circ} \mathrm{C}$, and $175^{\circ} \mathrm{C}$. Solid lines are measured data, dotted lines are calculations for a negative interface charge of $N_{i f}=3.2 \cdot 10^{12} \mathrm{~cm}^{-2}$ and a 2-DEG sheet charge of $N=10^{13} \mathrm{~cm}^{-2}$. Higher curves belong to higher temperatures both for measured and calculated data.
We have calculated several cases with different interface charge densities, while keeping $N=10^{13} \mathrm{~cm}^{-2}$, to find charge conditions that can explain the measured data. If the interface charge is varied, while $N$ is kept constant, a cross section of the transistor along the $y$-axis is in general not charge neutral. Compensating charges will reside elsewhere in the device apart from the 2-DEG and the interface. Our model calculations show that compensation charges that would reside deep in the device, e.g., at the GaN-substrate interface, will not influence the electric fields near the gate and therefore also not the Schottky current. In Fig. 10, we compare measurements at $25^{\circ} \mathrm{C}$ with calculations done with several charge configurations. Case 1 is the default negative interface charge density of $-3.2 \cdot 10^{12} \mathrm{~cm}^{-2}$ as also depicted in Fig. 9. The minus sign indicates that the net charge at the interface is negative. Case 2 has zero interface charge. Case 3 is with a positive interface charge of $10^{13} \mathrm{~cm}^{-2}$. Case 4 also has a positive interface charge of $10^{13} \mathrm{~cm}^{-2}$ for $x>5$ but a local, negative interface charge is assumed directly next to the gate between $0<x<5$, which corresponds to a distance of $125 \mathrm{~nm}$ adjacent to the gate. Case 5 has zero interface charge, like case 2 , but the gate angle (see Fig. 1) $\vartheta=90^{\circ}$ instead of $45^{\circ}$ for the other cases.

From Fig. 10, it is clear that the measurements can only be understood if there is a positive interface charge density that is larger than $10^{13} \mathrm{~cm}^{-2}$. This means that the interface charge would be comparable to the 2-DEG sheet charge. Although the transistor used in the measurements has a gate angle of $45^{\circ}$ considerable current increase will occur if the gate angle would be $90^{\circ}$, as can be seen comparing case 2 with case 5 . This is due to a sharper gate angle that directly leads to a higher electric field at the corner. In Fig. 11, the vertical electric field underneath the gate, $E_{T O T}(x)$, is plotted for case 2 at $-33.1 \mathrm{~V}$ and for case 5 at $-34.9 \mathrm{~V}$, so at comparable bias voltages. Note that the $x$-axis is on a logarithmic scale. $E_{\text {TOT }}(x)$ determines the Schottky current via (9). Near the gate corner at $x=0$, the field increase leads to a very rapid rise in current density. The increase of $E_{T O T}(x)$ near the

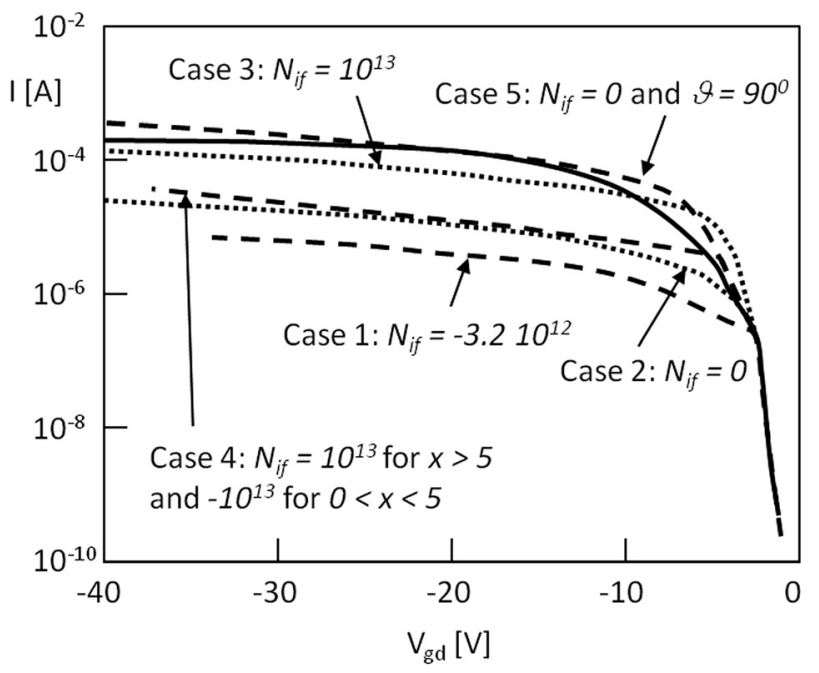

FIG. 10. Schottky current vs. voltage at $25^{\circ} \mathrm{C}$. Solid line is measured data. Dotted lines are calculations for several different interface charge configurations. 


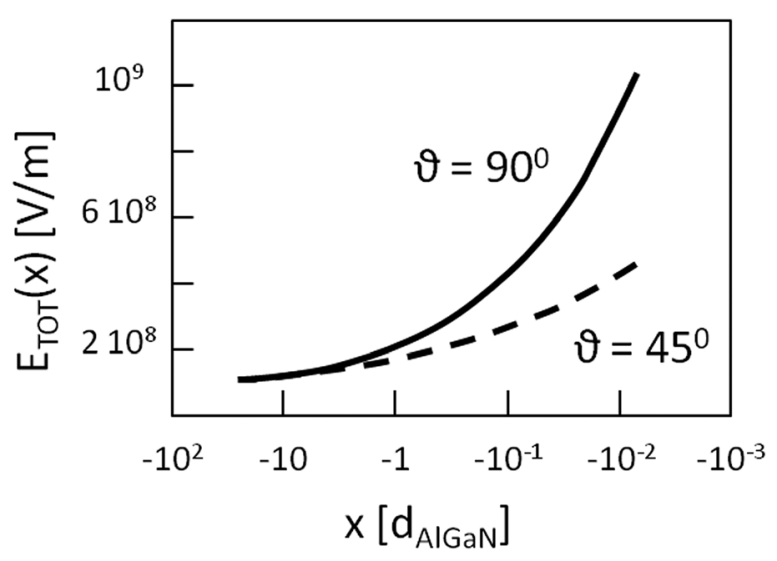

FIG. 11. Vertical electric field underneath the gate at $y=0$ as function of distance $\mathrm{x}$ to the gate corner for cases $2\left(45^{\circ}\right)$ and $5\left(90^{\circ}\right)$. Note that the $\mathrm{x}$ axis in on a logarithmic scale. The higher electric field for $90^{\circ}$ at the gate corner explains why case 5 has a much higher current than case 2 .

gate corner is much steeper for an angle of $90^{\circ}$ than for one of $45^{\circ}$. This explains the much higher current for case 5 compared to case 2 in Fig. 10. It underscores the importance of careful gate angle and field plate design. Note that our model only takes the gate angle but not any field plate into account.

Comparing cases 3 and 4 shows what happens if electrons from the gate are injected into traps at the interface next to the gate. ${ }^{13,14}$ The Schottky current would decrease because the electric fields at the gate edge are reduced by the negative interface charges. The hysteresis observed in Figs. 7 and 8 cannot be understood by this mechanism. On the contrary, the hysteresis seems to point to an increase of the positive interface charge due to high voltage stresses. This is in conflict with the proposed model in Ref. 1 in which a high drain voltage would lead to an increase of the electron concentration at the interface.

Comparing cases 1,2 , and 3 , it is clear that the current increases if the interface charge becomes more positive (or less negative). A more positive interface charge will also lead to more electrons in the 2-DEG and a higher maximum drain current and output power. Since GaN suppliers strive to increase the output power of the device, careful process optimization should be done to avoid an increase in gate leakage current.

\section{CONCLUSIONS}

A model has been derived for the gate current in a GaN HEMT device with a Schottky gate. At high reverse voltages below threshold, the current is increased due to high electric fields at the gate edge. A two-dimensional model is derived that calculates these fields and the subsequent current if the 2-DEG sheet charge density and the charge configuration at the semiconductor-passivation interface are given. Experiments show that voltage and temperature stresses change the charge configuration, such that measured drain-gate $I-V$ curves show hysteresis. These phenomena can be understood as a change in interface charge density. At high enough reverse bias, the gate current is dominated by Fowler-Nordheim tunneling, making the current almost independent of temperature and only slightly on voltage. The currents at low and at high bias are both determined by defects that have locally reduced the Schottky barrier height and that have a total surface area that is only a fraction $\left(10^{-3}\right.$ to $\left.10^{-4}\right)$ of the total gate contact area.

Reduction of the gate current can be obtained in two ways.

(1) By reduction of the number of defects or by increasing the defect barrier height. This route focusses on the epitaxial layer quality and the Schottky gate processing. Improvements should be visible by reduction of the current between threshold voltage and zero volt.

(2) By reducing the positive interface charge density. This can be done by improving the passivation layer processing. Such improvements will lead to less current increase with higher drain bias. Since it will generally also lead to less variation in interface charges, it will also improve hysteresis and memory effects in the drain-gate $I-V$ curves.

\section{ACKNOWLEDGMENTS}

The author likes to thank UMS, Ulm, Germany for providing the Schottky diodes and Fredrik Allerstam at NXP Semiconductors, Nijmegen, The Netherlands for the measurements and helpful discussions. This work was supported by the $\mathrm{GHz}$ Centre at Chalmers University, Gothenburg, Sweden.

\section{APPENDIX: CALCULATION OF THE POTENTIAL}

The potential of a sheet charge with density $\rho_{2 d}$ in the 2-DEG is given by (1b) where $r_{2 d}$ is given by

$r_{2 d}(x, y, t)=\sqrt{\left\{\left(x^{2}+y^{2}\right)^{\alpha / 2} \cos \left[\alpha \operatorname{atan}\left(\frac{y}{-x}\right)\right]-\left(t^{2}+1\right)^{\alpha / 2} \cos \left[\alpha \operatorname{atan}\left(\frac{-1}{t}\right)\right]\right\}^{2}+\left\{\left(x^{2}+y^{2}\right)^{\alpha / 2} \sin \left[\alpha \operatorname{atan}\left(\frac{y}{-x}\right)\right]-\left(t^{2}+1\right)^{\alpha / 2} \sin \left[\alpha \operatorname{atan}\left(\frac{-1}{t}\right)\right]\right\}^{2}}$

and $r^{\prime}{ }_{2 d}$ by

$r_{2 d}^{\prime}(x, y, t)=\sqrt{\left\{\left(x^{2}+y^{2}\right)^{\alpha / 2} \cos \left[\alpha \operatorname{atan}\left(\frac{y}{-x}\right)\right]-\left(t^{2}+1\right)^{\alpha / 2} \cos \left[\alpha \operatorname{atan}\left(\frac{-1}{t}\right)\right]\right\}^{2}+\left\{\left(x^{2}+y^{2}\right)^{\alpha / 2} \sin \left[\alpha \operatorname{atan}\left(\frac{y}{-x}\right)\right]+\left(t^{2}+1\right)^{\alpha / 2} \sin \left[\alpha \operatorname{atan}\left(\frac{-1}{t}\right)\right]\right\}^{2}}$. 
The real part of the potential is

$$
\operatorname{Re}\left[\Psi_{2 d}(x, y)\right]=\frac{\rho_{2 d} q d_{A l G a N}}{2 \pi \varepsilon} \int_{\Delta}^{\delta_{2 d}} \ln \left(\frac{r_{2 d}(x, y, t)}{r^{\prime}{ }_{2 d}(x, y, t)}\right) d t
$$

So $t$ is the integration variable for the 2-DEG charge from a position $\Delta$ that is far enough underneath the gate, where the current flow is one-dimensional, to its depletion boundary $\delta_{2 d}$. In practice, any value $\Delta \ll-1$ will suffice. The factor $d_{A l G a N}$ is a correction for taking the dimensions of the $z$-plane in units of the AlGaN thickness $d_{\text {AlGaN }}$.

The contribution of interface charges is calculated in an analogous way using

$$
\begin{aligned}
r_{i f}(x, y, t) & =\sqrt{\left\{\left(x^{2}+y^{2}\right)^{\alpha / 2} \cos \left[\alpha \operatorname{atan}\left(\frac{y}{-x}\right)\right]-t^{\alpha} \cos (\alpha \pi)\right\}^{2}+\left\{\left(x^{2}+y^{2}\right)^{\alpha / 2} \sin \left[\alpha \operatorname{atan}\left(\frac{y}{-x}\right)\right]-t^{\alpha} \sin (\alpha \pi)\right\}^{2}} \\
r_{i f}^{\prime}(x, y, t) & =\sqrt{\left\{\left(x^{2}+y^{2}\right)^{\alpha / 2} \cos \left[\alpha \operatorname{atan}\left(\frac{y}{-x}\right)\right]-t^{\alpha} \cos (\alpha \pi)\right\}^{2}+\left\{\left(x^{2}+y^{2}\right)^{\alpha / 2} \sin \left[\alpha \operatorname{atan}\left(\frac{y}{-x}\right)\right]+t^{\alpha} \sin (\alpha \pi)\right\}^{2}}
\end{aligned}
$$

and

$$
\operatorname{Re}\left[\Psi_{i f}(x, y)\right]=\frac{\rho_{i f} q d_{A l G a N}}{2 \pi \varepsilon} \int_{0}^{\delta_{g d}} \ln \left(\frac{r_{i f}(x, y, t)}{r^{\prime}{ }^{i f}(x, y, t)}\right) d t .
$$

It is easy to see how to extend (A1) and (A2) to include any arbitrary charge distribution in the 2-DEG and at the passivation interface, respectively. For example, to include also the charges of the partially depleted 2-DEG for $x>\delta_{2 d}$ as described by $\rho_{2 d}(x)$ in (3), we have to modify (A1) to

$$
\operatorname{Re}\left[\Psi_{2 d}(x, y)\right]=\frac{q d_{A l G a N}}{2 \pi \varepsilon} \int_{\Delta}^{d_{g d}} \rho_{2 d}(t) \ln \left(\frac{r_{2 d}(x, y, t)}{r_{2 d}^{\prime}(x, y, t)}\right) d t .
$$

Note the change in the upper integral boundary.

The drain contact is modeled by an additional charge $N_{d}$ in the 2-DEG uniformly distributed between $d_{g d}$ and $d_{g d}+\Delta_{d}$ and its contribution to the potential is

$$
\operatorname{Re}\left[\Psi_{\text {drain }}(x, y)\right]=\frac{N_{d} q d_{A l G a N}}{\Delta_{d} 2 \pi \varepsilon} \int_{d_{g d}}^{d_{g d}+\Delta_{d}} \ln \left(\frac{r_{2 d}(x, y, t)}{r^{\prime} 2 d(x, y, t)}\right) d t .
$$

$\Delta_{d}$ can be chosen rather arbitrarily since $N_{d}$ is used as a fitting parameter. We take $\Delta_{d}=20$. The total potential is simply the sum of (A2)-(A4).

${ }^{1}$ A. Goswami, R. J. Trew, and G. L. Bilbro, "Physics based modeling of gate leakage current due to traps in AlGaN/GaN HFETs," Solid State Electron. 80, 23-27 (2013).

${ }^{2} \mathrm{R}$. Jos, "Reverse Schottky gate current in AlGaN-GaN high-electron-mobility-transistors," J. Appl. Phys. 112, 094508 (2012).
${ }^{3}$ W. Saito, M. Kuraguchi, Y. Takada, K. Tsuda, I. Omura, and T. Ogura, "Influence of surface defect charge at AlGaN-GaN HEMT upon Schottky gate leakage current and breakdown voltage," IEEE Trans. Electron. Devices 52(2), 159-164 (2005).

${ }^{4} \mathrm{M}$. Hösch, "Analysis and optimization of AlGaN/GaN high electron mobility transistors for microwave applications," Ph.D. dissertation (University Ulm, Germany, 2011).

${ }^{5}$ K. Hayashi, Y. Yamaguchi, T. Oishi, H. Otsuka, K. Yamanaka, M. Nakayama, and Y. Miyamoto, "Mechanism study of gate leakage current for $\mathrm{AlGaN} / \mathrm{GaN}$ high electron mobility transistor structure under high reverse bias by thin surface barrier model and technology computer aided design simulation,” Jpn. J. Appl. Phys., Part 1 52, 04CF12 (2013).

${ }^{6}$ A. D. Wunsch, Complex Variables with Applications (Addison-Wesley Pub. Com., Reading, Massachusetts, 1994).

${ }^{7}$ G. B. Arfken and H. J. Weber, Mathematical Methods for Physicists, 5th ed. (Harcourt Academic, 2001).

${ }^{8}$ W. K. H. Panofsky and M. Phillips, Classical Electricity and Magnetism (Addison-Wesley Pub. Com., Reading, Massachusetts, 1975).

${ }^{9}$ J. P. Ibbetson, P. T. Fini, K. D. Ness, S. P. DenBaars, J. S. Speck, and U. K. Mishra, "Polarization effects, surface states, and the source of electrons in AlGaN/GaN heterostructure field effect transistors," Appl. Phys. Lett. 77(2), 250-252 (2000).

${ }^{10} \mathrm{~B}$. Jogai, "Influence of surface states on the two-dimensional electron gas in AlGaN/GaN heterojunction field-effect transistors," J. Appl. Phys. 93(3), 1631-1635 (2003).

${ }^{11}$ A. Ashok, D. Vasileska, S. M. Goodnick, and O. L. Hartin, "Importance of the gate-dependent polarization charge on the operation of GaN HEMTs," IEEE Trans. Electron. Devices 56(5), 998-1005 (2009).

${ }^{12}$ O. Ambacher, J. Smart, J. R. Shealy, N. G. Weimann, K. Chu, M. Murphy, W. J. Schaff, L. F. Eastman, R. Dimitrov, L. Wittmer, M. Stutzman, W. Rieger, and J. Hilsenbeck, "Two-dimensional electron gases induced by spontaneous and piezoelectric polarization charges in N- and Ga-face AlGaN/GaN heterostructures," J. Appl. Phys. 85(6), 3222-3233 (1999).

${ }^{13}$ H. Yin, G. L. Bilbro, and R. J. Trew, "A new physics-based compact model for AlGaN/Gan HFETs," in IEEE/MTT-S, Microwave Symposium (2007), pp. 787-790.

${ }^{14}$ S. Sabuktagin, Y.-T. Moon, S. Dogan, A. A. Baski, and H. Morkoç, "Observation of surface charging at the edge of a Schottky contact," IEEE Electron Dev. Lett. 27(4), 211-213 (2006). 